\title{
A characterization of nonhomogeneous wavelet dual frames in Sobolev spaces
}

\author{
Jian-Ping Zhang* and Yun-Zhang Li
}

\section{"Correspondence:}

zhjp1982@emails.bjut.edu.cn

College of Applied Sciences, Beijing

University of Technology, Beijing,

100124, China

\begin{abstract}
In recent years, nonhomogeneous wavelet frames have attracted some mathematicians' interest. This paper investigates such problems in a Sobolev space setting. A characterization of nonhomogeneous wavelet dual frames in Sobolev spaces pairs is obtained.
\end{abstract}

MSC: $42 \mathrm{C} 40 ; 42 \mathrm{C} 15$

Keywords: Bessel sequence; frame; nonhomogeneous wavelet dual frame; Sobolev spaces

\section{Introduction}

Wavelet frames in $L^{2}\left(\mathbb{R}^{d}\right)$ have been widely investigated by many authors [1-8]. In particular, homogeneous wavelet dual frames in $L^{2}\left(\mathbb{R}^{d}\right)$ were first characterized by Han [9], and then studied by Bownik [3]. For homogeneous wavelet dual frames, regularity and vanishing moments have been both required. However, for nonhomogeneous wavelet dual frames in Sobolev space pairs $\left(H^{s}\left(\mathbb{R}^{d}\right), H^{-s}\left(\mathbb{R}^{d}\right)\right)$, they can be separated. It makes it easy to construct dual frames (see [10-14] for details). This paper is devoted to characterizing nonhomogeneous wavelet dual frames in Sobolev spaces pairs $\left(H^{s}\left(\mathbb{R}^{d}\right), H^{-s}\left(\mathbb{R}^{d}\right)\right)$ via a pair of equations.

Before proceeding, we introduce some notions and notations. We denote by $\mathbb{Z}$ and $\mathbb{N}$ the set of integers and the set of positive integers, respectively. Let $d \in \mathbb{N}$. We denote by $\mathbb{T}^{d}=[0,1)^{d}$ the $d$-dimensional torus. For a Lebesgue measurable set $E$ in $\mathbb{R}^{d}$, we denote by $|E|$ its Lebesgue measure and $\chi_{E}$ the characteristic function of $E$, respectively. And we write $\delta$ for the Dirac sequence, i.e., $\delta_{0,0}=1$ and $\delta_{0, k}=0$ for $0 \neq k \in \mathbb{Z}^{d}$. The Fourier transform of a function $f \in L^{1}\left(\mathbb{R}^{d}\right) \cap L^{2}\left(\mathbb{R}^{d}\right)$ is defined by

$$
\hat{f}(\cdot)=\int_{\mathbb{R}^{d}} f(x) e^{-2 \pi i(x, \cdot)} d x,
$$

and extended to $L^{2}\left(\mathbb{R}^{d}\right)$ as usual, where $\langle\cdot, \cdot\rangle$ denotes the Euclidean inner product in $\mathbb{R}^{d}$.

For $s \in \mathbb{R}$, we define Sobolev spaces $H^{s}\left(\mathbb{R}^{d}\right)$ as the space of all tempered distributions $f$ such that

$$
\|f\|_{H^{s}\left(\mathbb{R}^{d}\right)}^{2}=\int_{\mathbb{R}^{d}}|\hat{f}(\xi)|^{2}\left(1+\|\xi\|^{2}\right)^{s} d \xi<\infty,
$$

(c) Zhang and Li 2016. This article is distributed under the terms of the Creative Commons Attribution 4.0 International License (http://creativecommons.org/licenses/by/4.0/), which permits unrestricted use, distribution, and reproduction in any medium, provided you give appropriate credit to the original author(s) and the source, provide a link to the Creative Commons license, and indicate if changes were made. 
where $\|\cdot\|$ denotes the Euclidean norm on $\mathbb{R}^{d}$. The inner product in $H^{s}\left(\mathbb{R}^{d}\right)$ is given by

$$
\langle f, g\rangle_{H^{s}\left(\mathbb{R}^{d}\right)}=\int_{\mathbb{R}^{d}} \hat{f}(\xi) \overline{\hat{g}(\xi)}\left(1+\|\xi\|^{2}\right)^{s} d \xi, \quad f, g \in H^{s}\left(\mathbb{R}^{d}\right) .
$$

Moreover, for each $g \in H^{-s}\left(\mathbb{R}^{d}\right)$,

$$
\langle f, g\rangle=\int_{\mathbb{R}^{d}} \hat{f}(\xi) \overline{\hat{g}(\xi)} d \xi, \quad f \in H^{s}\left(\mathbb{R}^{d}\right)
$$

is a linear continuous functional in $H^{s}\left(\mathbb{R}^{d}\right)$. The $H^{s}\left(\mathbb{R}^{d}\right)$ and $H^{-s}\left(\mathbb{R}^{d}\right)$ form pairs of dual spaces.

For functions $f, g: \mathbb{R}^{d} \mapsto \mathbb{C}$, define

$$
[f, g]_{t}(\cdot)=\sum_{k \in \mathbb{Z}^{d}} f(\cdot+k) \overline{g(\cdot+k)}\left(1+\|\cdot+k\|^{2}\right)^{t}, \quad t \in \mathbb{R} .
$$

For convenience, we write

$$
f_{j, k}(\cdot)=2^{\frac{j d}{2}} f\left(2^{j} \cdot-k\right) \quad \text { and } \quad f_{j, k}^{s}(\cdot)=2^{j\left(\frac{d}{2}-s\right)} f\left(2^{j} \cdot-k\right)
$$

for a distribution $f, j \in \mathbb{Z}, k \in \mathbb{Z}^{d}$, and $s \in \mathbb{R}$.

Let $\mathbb{N}_{0}=\mathbb{N} \cup\{0\}$. Given $L \in \mathbb{N}$ and $s \in \mathbb{R}$, let $\phi, \psi_{1}, \psi_{2}, \ldots, \psi_{L} \in H^{s}\left(\mathbb{R}^{d}\right)$ and $\tilde{\phi}, \tilde{\psi}_{1}, \tilde{\psi}_{2}, \ldots$, $\tilde{\psi}_{L} \in H^{-s}\left(\mathbb{R}^{d}\right)$, we denote by $X^{s}\left(\phi ; \psi_{1}, \psi_{2}, \ldots, \psi_{L}\right)$ and $X^{-s}\left(\tilde{\phi} ; \tilde{\psi}_{1}, \tilde{\psi}_{2}, \ldots, \tilde{\psi}_{L}\right)$ the following two nonhomogeneous wavelet systems in $H^{s}\left(\mathbb{R}^{d}\right)$ and $H^{-s}\left(\mathbb{R}^{d}\right)$, respectively:

$$
X^{s}\left(\phi ; \psi_{1}, \psi_{2}, \ldots, \psi_{L}\right)=\left\{\phi_{0, k}: k \in \mathbb{Z}^{d}\right\} \cup\left\{\psi_{l, j, k}^{s}: j \in \mathbb{N}_{0}, k \in \mathbb{Z}^{d}, l=1,2, \ldots, L\right\}
$$

and

$$
X^{-s}\left(\tilde{\phi} ; \tilde{\psi}_{1}, \tilde{\psi}_{2}, \ldots, \tilde{\psi}_{L}\right)=\left\{\tilde{\phi}_{0, k}: k \in \mathbb{Z}^{d}\right\} \cup\left\{\tilde{\psi}_{l, j, k}^{-s}: j \in \mathbb{N}_{0}, k \in \mathbb{Z}^{d}, l=1,2, \ldots, L\right\}
$$

We say that $X^{s}\left(\phi ; \psi_{1}, \psi_{2}, \ldots, \psi_{L}\right)$ is a nonhomogeneous wavelet frame in $H^{s}\left(\mathbb{R}^{d}\right)$ if there exist two positive constants $A, B$ such that

$$
\begin{aligned}
A\|f\|_{H^{s}\left(\mathbb{R}^{d}\right)}^{2} & \leq \sum_{k \in \mathbb{Z}^{d}}\left|\left\langle f, \phi_{0, k}\right\rangle_{H^{s}\left(\mathbb{R}^{d}\right)}\right|^{2}+\sum_{l=1}^{L} \sum_{j=0}^{\infty} \sum_{k \in \mathbb{Z}^{d}}\left|\left\langle f, \psi_{l, j, k}^{s}\right\rangle_{H^{s}\left(\mathbb{R}^{d}\right)}\right|^{2} \\
& \leq B\|f\|_{H^{s}\left(\mathbb{R}^{d}\right)}^{2}, \quad \forall f \in H^{s}\left(\mathbb{R}^{d}\right),
\end{aligned}
$$

where $A, B$ are called frame bounds; it is called a nonhomogeneous wavelet Bessel sequence in $H^{s}\left(\mathbb{R}^{d}\right)$ if the right-hand inequality in (1.3) holds, where $B$ is called a Bessel bound. Furthermore, we say that $\left(X^{s}\left(\phi ; \psi_{1}, \psi_{2}, \ldots, \psi_{L}\right), X^{-s}\left(\tilde{\phi} ; \tilde{\psi}_{1}, \tilde{\psi}_{2}, \ldots, \tilde{\psi}_{L}\right)\right)$ is a pair of nonhomogeneous wavelet dual frames in $\left(H^{s}\left(\mathbb{R}^{d}\right), H^{-s}\left(\mathbb{R}^{d}\right)\right)$ if $X^{s}\left(\phi ; \psi_{1}, \psi_{2}, \ldots, \psi_{L}\right)$ and $X^{-s}\left(\tilde{\phi} ; \tilde{\psi}_{1}, \tilde{\psi}_{2}, \ldots, \tilde{\psi}_{L}\right)$ are Bessel sequences in $H^{s}\left(\mathbb{R}^{d}\right)$ and $H^{-s}\left(\mathbb{R}^{d}\right)$, respectively, and

$$
\langle f, g\rangle=\sum_{k \in \mathbb{Z}^{d}}\left\langle f, \tilde{\phi}_{0, k}\right\rangle\left\langle\phi_{0, k}, g\right\rangle+\sum_{l=1}^{L} \sum_{j=0}^{\infty} \sum_{k \in \mathbb{Z}^{d}}\left\langle f, \tilde{\psi}_{l, j, k}^{-s}\right\rangle\left\langle\psi_{l, j, k}^{s}, g\right\rangle
$$

holds for all $f \in H^{s}\left(\mathbb{R}^{d}\right)$ and $g \in H^{-s}\left(\mathbb{R}^{d}\right)$. 
If $\left(X^{s}\left(\phi ; \psi_{1}, \psi_{2}, \ldots, \psi_{L}\right), X^{-s}\left(\tilde{\phi} ; \tilde{\psi}_{1}, \tilde{\psi}_{2}, \ldots, \tilde{\psi}_{L}\right)\right)$ is a pair of dual frames in $\left(H^{s}\left(\mathbb{R}^{d}\right)\right.$, $\left.H^{-s}\left(\mathbb{R}^{d}\right)\right)$, then it follows from (1.4) that

$$
f=\sum_{k \in \mathbb{Z}^{d}}\left\langle f, \tilde{\phi}_{0, k}\right\rangle \phi_{0, k}+\sum_{l=1}^{L} \sum_{j=0}^{\infty} \sum_{k \in \mathbb{Z}^{d}}\left\langle f, \tilde{\psi}_{l, j, k}^{-s}\right\rangle \psi_{l, j, k}^{s}, \quad f \in H^{s}\left(\mathbb{R}^{d}\right),
$$

and

$$
g=\sum_{k \in \mathbb{Z}^{d}}\left\langle g, \phi_{0, k}\right\rangle \tilde{\phi}_{0, k}+\sum_{l=1}^{L} \sum_{j=0}^{\infty} \sum_{k \in \mathbb{Z}^{d}}\left\langle g, \psi_{l, j, k}^{s}\right) \tilde{\psi}_{l, j, k}^{-s}, \quad g \in H^{-s}\left(\mathbb{R}^{d}\right),
$$

with the series converging unconditionally in $H^{s}\left(\mathbb{R}^{d}\right)$ and $H^{-s}\left(\mathbb{R}^{d}\right)$, respectively.

The paper is organized as follows. Section 2 is devoted to some lemmas used latter. Section 3 is devoted to characterizing nonhomogeneous wavelet dual frames in $\left(H^{s}\left(\mathbb{R}^{d}\right), H^{-s}\left(\mathbb{R}^{d}\right)\right)$ via a pair of equations.

\section{Some lemmas}

In this section, we give some auxiliary lemmas which are necessary in proving Theorem 3.1 below.

Definition 2.1 Define a function $\kappa: \mathbb{Z}^{d} \rightarrow \mathbb{Z}$ by

$$
\kappa(n)=\sup \left\{j \geq 0: 2^{-j} n \in \mathbb{Z}^{d}\right\}
$$

for $0 \neq n \in \mathbb{Z}^{d}$, and set $\kappa(0)=+\infty$.

Lemma 2.1 Let $s \in \mathbb{R}, j \in \mathbb{Z}$, and $\psi \in H^{-s}\left(\mathbb{R}^{d}\right)$. Then, for $f \in H^{s}\left(\mathbb{R}^{d}\right)$ and $k \in \mathbb{Z}^{d}$, the $k$ th Fourier coefficient of $\left[2^{\frac{j d}{2}} \hat{f}\left(2^{j} \cdot\right), \hat{\psi}(\cdot)\right]_{0}(\xi)$ is $\left\langle f, \psi_{j, k}\right\rangle$. In particular,

$$
\left[2^{\frac{j d}{2}} \hat{f}\left(2^{j} \cdot\right), \hat{\psi}(\cdot)\right]_{0}(\xi)=\sum_{k \in \mathbb{Z}^{d}}\left\langle f, \psi_{j, k}\right\rangle e^{2 \pi i\langle k, \xi\rangle}
$$

if $\left\{\psi_{j, k}: k \in \mathbb{Z}^{d}\right\}$ is a Bessel sequence in $H^{-s}\left(\mathbb{R}^{d}\right)$.

Proof Since $f \in H^{s}\left(\mathbb{R}^{d}\right)$ and $\psi \in H^{-s}\left(\mathbb{R}^{d}\right)$, we have $\hat{f}\left(2^{j} \cdot\right) \overline{\hat{\psi}(\cdot)} \in L^{1}\left(\mathbb{R}^{d}\right)$, and thus

$$
\begin{aligned}
\int_{\mathbb{T}^{d}}\left[2^{\frac{j d}{2}} \hat{f}\left(2^{j} \cdot\right), \hat{\psi}(\cdot)\right]_{0}(\xi) e^{-2 \pi i\langle k, \xi\rangle} d \xi & =2^{\frac{j d}{2}} \int_{\mathbb{T}^{d}} \sum_{l \in \mathbb{Z}^{d}} \hat{f}\left(2^{j}(\xi+l)\right) \overline{\hat{\psi}(\xi+l)} e^{-2 \pi i\langle k, \xi\rangle} d \xi \\
& =2^{\frac{j d}{2}} \int_{\mathbb{R}^{d}} \hat{f}\left(2^{j} \xi\right) \overline{\hat{\psi}(\xi)} e^{-2 \pi i\langle k, \xi\rangle} d \xi \\
& =2^{-\frac{j d}{2}} \int_{\mathbb{R}^{d}} \hat{f}(\xi) \overline{\hat{\psi}\left(2^{-j \xi}\right)} e^{-2 \pi i\left\langle k, 2^{-j} \xi\right\rangle} d \xi \\
& =\int_{\mathbb{R}^{d}} \hat{f}(\xi) \overline{\left[\psi_{j, k}(\cdot)\right]^{\wedge}(\xi)} d \xi,
\end{aligned}
$$

by the Plancherel theorem. So

$$
\int_{\mathbb{T}^{d}}\left[2^{\frac{j d}{2}} \hat{f}\left(2^{j} \cdot\right), \hat{\psi}(\cdot)\right]_{0}(\xi) e^{-2 \pi i\langle k, \xi\rangle} d \xi=\left\langle f, \psi_{j, k}\right\rangle .
$$


If $\left\{\psi_{j, k}: k \in \mathbb{Z}^{d}\right\}$ is a Bessel sequence in $H^{-s}\left(\mathbb{R}^{d}\right)$, then $\left\{\left\langle f, \psi_{j, k}\right\rangle\right\}_{k \in \mathbb{Z}^{d}} \in \ell^{2}\left(\mathbb{Z}^{d}\right)$, and thus (2.1) follows by (2.2).

By a careful observation of the proof of [13], Proposition 2.1, we have the following.

Lemma 2.2 Let $s \in \mathbb{R}, \phi, \psi_{1}, \psi_{2}, \ldots, \psi_{L} \in H^{s}\left(\mathbb{R}^{d}\right)$. Then $X^{s}\left(\phi ; \psi_{1}, \psi_{2}, \ldots, \psi_{L}\right)$ is a Bessel sequence in $H^{s}\left(\mathbb{R}^{d}\right)$ with Bessel bound $B$ if and only if

$$
\sum_{k \in \mathbb{Z}^{d}}\left|\left\langle g, \phi_{0, k}\right\rangle\right|^{2}+\sum_{l=1}^{L} \sum_{j=0}^{\infty} \sum_{k \in \mathbb{Z}^{d}}\left|\left\langle g, \psi_{l, j, k}^{s}\right\rangle\right|^{2} \leq B\|g\|_{H^{-s}\left(\mathbb{R}^{d}\right)}^{2} \quad \text { for } g \in H^{-s}\left(\mathbb{R}^{d}\right) .
$$

Lemma 2.3 Let $s \in \mathbb{R}, \phi, \psi_{1}, \psi_{2}, \ldots, \psi_{L} \in H^{s}\left(\mathbb{R}^{d}\right)$. Suppose that $X^{s}\left(\phi ; \psi_{1}, \psi_{2}, \ldots, \psi_{L}\right)$ is a Bessel sequence in $H^{s}\left(\mathbb{R}^{d}\right)$ with Bessel bound $B$, then

$$
|\hat{\phi}(\cdot)|^{2}+\sum_{l=1}^{L} \sum_{j=0}^{\infty} 2^{-2 j s}\left|\hat{\psi}_{l}\left(2^{-j} \cdot\right)\right|^{2} \leq B\left(1+\|\cdot\|^{2}\right)^{-s}
$$

holds a.e. on $\mathbb{R}^{d}$.

Proof Since $X^{s}\left(\phi ; \psi_{1}, \psi_{2}, \ldots, \psi_{L}\right)$ is a Bessel sequence in $H^{s}\left(\mathbb{R}^{d}\right)$ with Bessel bound $B$, by Lemma 2.2, we have

$$
\sum_{k \in \mathbb{Z}^{d}}\left|\left\langle g, \phi_{0, k}\right\rangle\right|^{2}+\sum_{l=1}^{L} \sum_{j=0}^{\infty} \sum_{k \in \mathbb{Z}^{d}}\left|\left\langle g, \psi_{l, j, k}^{s}\right\rangle\right|^{2} \leq B\|g\|_{H^{-s}\left(\mathbb{R}^{d}\right)}^{2} \quad \text { for } g \in H^{-s}\left(\mathbb{R}^{d}\right) .
$$

By Lemma 2.1 and an argument similar to that of [6], Theorem 1, we get

$$
\begin{aligned}
\sum_{k \in \mathbb{Z}^{d}}\left|\left\langle g, \phi_{0, k}\right\rangle\right|^{2}+\sum_{l=1}^{L} \sum_{j=0}^{\infty} \sum_{k \in \mathbb{Z}^{d}}|| g,\left.\psi_{l, j, k}^{s}\right|^{2} \\
=\int_{\mathbb{R}^{d}} \hat{\phi}(\xi) \overline{\hat{g}(\xi)} \sum_{k \in \mathbb{Z}^{d}} \hat{g}(\xi+k) \overline{\hat{\phi}(\xi+k)} d \xi \\
\quad+\sum_{l=1}^{L} \sum_{j=0}^{\infty} 2^{-2 j s} \int_{\mathbb{R}^{d}} \hat{\psi}_{l}\left(2^{-j} \xi\right) \overline{\hat{g}(\xi)} \sum_{k \in \mathbb{Z}^{d}} \hat{g}\left(\xi+2^{j} k\right) \overline{\hat{\psi}_{l}\left(2^{-j} \xi+k\right)} d \xi .
\end{aligned}
$$

It can be rewritten as

$$
\begin{aligned}
& \sum_{k \in \mathbb{Z}^{d}}\left|\left\langle g, \phi_{0, k}\right\rangle\right|^{2}+\sum_{l=1}^{L} \sum_{j=0}^{\infty} \sum_{k \in \mathbb{Z}^{d}}\left|\left\langle g, \psi_{l, j, k}^{s}\right\rangle\right|^{2} \\
& =\int_{\mathbb{R}^{d}}|\hat{g}(\xi)|^{2}\left(|\hat{\phi}(\xi)|^{2}+\sum_{l=1}^{L} \sum_{j=0}^{\infty} 2^{-2 j s}\left|\hat{\psi}_{l}\left(2^{-j} \xi\right)\right|^{2}\right) d \xi \\
& \quad+\int_{\mathbb{R}^{d}} \frac{\hat{g}(\xi)}{\sum_{0 \neq k \in \mathbb{Z}^{d}} \hat{g}(\xi+k)} \\
& \quad \times\left(\hat{\phi}(\xi) \overline{\hat{\phi}(\xi+k)}+\sum_{l=1}^{L} \sum_{j=0}^{\kappa(k)} 2^{-2 j s} \hat{\psi}_{l}\left(2^{-j} \xi\right) \overline{\hat{\psi}_{l}\left(2^{-j}(\xi+k)\right)}\right) d \xi
\end{aligned}
$$

by the definition of $\kappa$. 
Suppose (2.4) does not hold. Then there exists $E \subset \mathbb{R}^{d}$ with $|E|>0$ such that

$$
|\hat{\phi}(\cdot)|^{2}+\sum_{l=1}^{L} \sum_{j=0}^{\infty} 2^{-2 j s}\left|\hat{\psi}_{l}\left(2^{-j} \cdot\right)\right|^{2}>B\left(1+\|\cdot\|^{2}\right)^{-s} \text { on } E,
$$

and thus

$$
|\hat{\phi}(\cdot)|^{2}+\sum_{l=1}^{L} \sum_{j=0}^{\infty} 2^{-2 j s}\left|\hat{\psi}_{l}\left(2^{-j}\right)\right|^{2}>B\left(1+\|\cdot\|^{2}\right)^{-s}
$$

on some $E^{\prime}=E \cap\left([0,1)^{d}+k_{0}\right)$ with $\left|E^{\prime}\right|>0$ and $k_{0} \in \mathbb{Z}^{d}$. Take $g$ such that $\hat{g}(\cdot)=(1+$ $\left.\|\cdot\|^{2}\right)^{s / 2} \chi_{E^{\prime}}$ in (2.6), then we obtain

$$
\left.\sum_{k \in \mathbb{Z}^{d}}\left|\left\langle g, \phi_{0, k}\right\rangle\right|^{2}+\sum_{l=1}^{L} \sum_{j=0}^{\infty} \sum_{k \in \mathbb{Z}^{d}}|| g, \psi_{l, j, k}^{s}\right\rangle\left.\right|^{2}>B\left|E^{\prime}\right|=B\|g\|_{H^{-s}\left(\mathbb{R}^{d}\right)}^{2},
$$

contradicting (2.5).

\section{The characterization of nonhomogeneous wavelet dual frames in Sobolev spaces}

This section is devoted to characterizing nonhomogeneous wavelet dual frames in $\left(H^{s}\left(\mathbb{R}^{d}\right), H^{-s}\left(\mathbb{R}^{d}\right)\right)$. The following theorem provides us with a characterization via a pair of equations.

Theorem 3.1 Let $s \in \mathbb{R}, \phi, \psi_{1}, \psi_{2}, \ldots, \psi_{L} \in H^{s}\left(\mathbb{R}^{d}\right)$ and $\tilde{\phi}, \tilde{\psi}_{1}, \tilde{\psi}_{2}, \ldots, \tilde{\psi}_{L} \in H^{-s}\left(\mathbb{R}^{d}\right)$. Define wavelet systems $X^{s}\left(\phi ; \psi_{1}, \psi_{2}, \ldots, \psi_{L}\right)$ and $X^{-s}\left(\tilde{\phi} ; \tilde{\psi}_{1}, \tilde{\psi}_{2}, \ldots, \tilde{\psi}_{L}\right)$ as in (1.1) and (1.2), respectively. Suppose that $X^{s}\left(\phi ; \psi_{1}, \psi_{2}, \ldots, \psi_{L}\right)$ is a Bessel sequence in $H^{s}\left(\mathbb{R}^{d}\right)$, and $X^{-s}\left(\tilde{\phi} ; \tilde{\psi}_{1}, \ldots\right.$, $\left.\tilde{\psi}_{L}\right)$ is a Bessel sequence in $H^{-s}\left(\mathbb{R}^{d}\right)$. Then $\left(X^{s}\left(\phi ; \psi_{1}, \psi_{2}, \ldots, \psi_{L}\right), X^{-s}\left(\tilde{\phi} ; \tilde{\psi}_{1}, \tilde{\psi}_{2}, \ldots, \tilde{\psi}_{L}\right)\right)$ is a pair of dual frames in $\left(H^{s}\left(\mathbb{R}^{d}\right), H^{-s}\left(\mathbb{R}^{d}\right)\right)$ if and only if, for every $k \in \mathbb{Z}^{d}$,

$$
\hat{\phi}(\cdot) \overline{\hat{\tilde{\phi}}(\cdot+k)}+\sum_{l=1}^{L} \sum_{j=0}^{\kappa(k)} \hat{\psi}_{l}\left(2^{-j} \cdot\right) \overline{\tilde{\tilde{\psi}}_{l}\left(2^{-j}(\cdot+k)\right)}=\delta_{0, k} \quad \text { a.e. on } \mathbb{R}^{d} .
$$

Proof By the definition, $\left(X^{s}\left(\phi ; \psi_{1}, \psi_{2}, \ldots, \psi_{L}\right), X^{-s}\left(\tilde{\phi} ; \tilde{\psi}_{1}, \tilde{\psi}_{2}, \ldots, \tilde{\psi}_{L}\right)\right)$ is a pair of dual frames for $\left(H^{s}\left(\mathbb{R}^{d}\right), H^{-s}\left(\mathbb{R}^{d}\right)\right)$ if and only if

$$
\begin{aligned}
& \sum_{k \in \mathbb{Z}^{d}}\left\langle f, \tilde{\phi}_{0, k}\right\rangle\left\langle\phi_{0, k}, g\right\rangle+\sum_{l=1}^{L} \sum_{j=0}^{\infty} \sum_{k \in \mathbb{Z}^{d}}\left\langle f, \tilde{\psi}_{l, j, k}^{-s}\right\rangle\left\langle\psi_{l, j, k}^{s}, g\right\rangle \\
& =\langle f, g\rangle, \quad f \in H^{s}\left(\mathbb{R}^{d}\right), g \in H^{-s}\left(\mathbb{R}^{d}\right) .
\end{aligned}
$$

By the Plancherel theorem and Lemma 2.1, we deduce that

$$
\begin{aligned}
& \sum_{k \in \mathbb{Z}^{d}}\langle f, \tilde{\phi}(\cdot-k)\rangle\langle\phi(\cdot-k), g\rangle+\sum_{l=1}^{L} \sum_{j=0}^{\infty} \sum_{k \in \mathbb{Z}^{d}}\left\langle f, \tilde{\psi}_{l, j, k}^{-s}\right\rangle\left\langle\psi_{l, j, k}^{s}, g\right\rangle \\
& =\int_{\mathbb{T}^{d}}\left(\sum_{k \in \mathbb{Z}^{d}} \hat{f}(\xi+k) \overline{\hat{\tilde{\phi}}(\xi+k)}\right)\left(\sum_{k \in \mathbb{Z}^{d}} \hat{\phi}(\xi+k) \overline{\hat{g}(\xi+k)}\right) d \xi
\end{aligned}
$$




$$
\begin{aligned}
& +\sum_{l=1}^{L} \sum_{j=0}^{\infty} 2^{j d} \int_{\mathbb{T}^{d}}\left(\sum_{k \in \mathbb{Z}^{d}} \hat{f}\left(2^{j}(\xi+k)\right) \overline{\hat{\tilde{\psi}}_{l}(\xi+k)}\right)\left(\sum_{k \in \mathbb{Z}^{d}} \hat{\psi}_{l}(\xi+k) \overline{\hat{g}(2 j(\xi+k))}\right) d \xi \\
& =\int_{\mathbb{R}^{d}} \sum_{k \in \mathbb{Z}^{d}} \hat{f}(\xi+k) \overline{\hat{\tilde{\phi}}(\xi+k)} \hat{\phi}(\xi) \overline{\hat{g}(\xi)} d \xi \\
& +\sum_{l=1}^{L} \sum_{j=0}^{\infty} 2^{j d} \int_{\mathbb{R}^{d}} \sum_{k \in \mathbb{Z}^{d}} \hat{f}\left(2^{j}(\xi+k)\right) \overline{\tilde{\tilde{\psi}}_{l}(\xi+k)} \hat{\psi}_{l}(\xi) \overline{\hat{g}\left(2^{j} \xi\right)} d \xi \\
& =\int_{\mathbb{R}^{d}} \hat{f}(\xi) \overline{\hat{g}(\xi)}\left(\hat{\phi}(\xi) \overline{\hat{\tilde{\phi}}(\xi)}+\sum_{l=1}^{L} \sum_{j=0}^{\infty} \hat{\psi}_{l}\left(2^{-j} \xi\right) \overline{\tilde{\tilde{\psi}}_{l}\left(2^{-j} \xi\right)}\right) d \xi \\
& +\int_{\mathbb{R}^{d}} \overline{\hat{g}(\xi)}\left(\sum_{0 \neq k \in \mathbb{Z}^{d}} \hat{f}(\xi+k) \hat{\phi}(\xi) \overline{\tilde{\hat{\phi}}(\xi+k)}\right. \\
& \left.+\sum_{l=1}^{L} \sum_{j=0}^{\infty} \sum_{0 \neq k \in \mathbb{Z}^{d}} \hat{f}\left(\xi+2^{j} k\right) \hat{\psi}_{l}\left(2^{-j} \xi\right) \overline{\hat{\tilde{\psi}}_{l}\left(2^{-j} \xi+k\right)}\right) d \xi \\
& =\int_{\mathbb{R}^{d}} \hat{f}(\xi) \overline{\hat{g}(\xi)}\left(\hat{\phi}(\xi) \overline{\hat{\tilde{\phi}}(\xi)}+\sum_{l=1}^{L} \sum_{j=0}^{\infty} \hat{\psi}_{l}\left(2^{-j} \xi\right) \overline{\tilde{\tilde{\psi}}_{l}\left(2^{-j} \xi\right)}\right) d \xi \\
& +\int_{\mathbb{R}^{d}} \overline{\hat{g}(\xi)} \sum_{0 \neq k \in \mathbb{Z}^{d}} \hat{f}(\xi+k)\left(\hat{\phi}(\xi) \overline{\hat{\tilde{\phi}}(\xi+k)}+\sum_{l=1}^{L} \sum_{j=0}^{\kappa(k)} \hat{\psi}_{l}\left(2^{-j} \xi\right) \overline{\tilde{\tilde{\psi}}_{l}\left(2^{-j}(\xi+k)\right)}\right) d \xi .
\end{aligned}
$$

And thus (3.2) can be rewritten as

$$
\begin{aligned}
& \int_{\mathbb{R}^{d}} \hat{f}(\xi) \overline{\hat{g}(\xi)}\left(\hat{\phi}(\xi) \overline{\tilde{\hat{\phi}}(\xi)}+\sum_{l=1}^{L} \sum_{j=0}^{\infty} \hat{\psi}_{l}\left(2^{-j} \xi\right) \overline{\tilde{\tilde{\psi}}_{l}\left(2^{-j} \xi\right)}\right) d \xi \\
& \quad+\int_{\mathbb{R}^{d}} \overline{\hat{g}(\xi)} \sum_{0 \neq k \in \mathbb{Z}^{d}} \hat{f}(\xi+k)\left(\hat{\phi}(\xi) \overline{\tilde{\hat{\phi}}(\xi+k)}+\sum_{l=1}^{L} \sum_{j=0}^{\kappa(k)} \hat{\psi}_{l}\left(2^{-j} \xi\right) \overline{\hat{\tilde{\psi}}_{l}\left(2^{-j}(\xi+k)\right)}\right) d \xi \\
& =\int_{\mathbb{R}^{d}} \hat{f}(\xi) \overline{\hat{g}(\xi)} d \xi
\end{aligned}
$$

Obviously, (3.1) implies (3.3). To finish the proof, next we prove the converse implication.

Suppose (3.3) holds. By Lemma 2.3 and the Cauchy-Schwarz inequality, the series

$$
\hat{\phi}(\cdot) \overline{\tilde{\tilde{\phi}}(\cdot+k)}+\sum_{l=1}^{L} \sum_{j=0}^{\kappa(k)} \hat{\psi}_{l}\left(2^{-j} \cdot\right) \overline{\tilde{\tilde{\psi}}_{l}\left(2^{-j}(\cdot+k)\right)}
$$

with $k \in \mathbb{Z}^{d}$ converges absolutely a.e. on $\mathbb{R}^{d}$ and belongs to $L^{\infty}\left(\mathbb{R}^{d}\right)$, and almost all points in $\mathbb{R}^{d}$ are Lebesgue points. Let $\xi_{0} \in \mathbb{R}^{d}$ be such a point. For $0<\epsilon<\frac{1}{2}$, take $f$ and $g$ such that

$$
\hat{f}(\cdot)=\frac{\left(1+\|\cdot\|^{2}\right)^{-s / 2} \chi_{B\left(\xi_{0}, \epsilon\right)}}{\sqrt{\left|B\left(\xi_{0}, \epsilon\right)\right|}} \text { and } \hat{g}(\cdot)=\frac{\left(1+\|\cdot\|^{2}\right)^{s / 2} \chi_{B\left(\xi_{0}, \epsilon\right)}}{\sqrt{\left|B\left(\xi_{0}, \epsilon\right)\right|}}
$$


in (3.3), where $B\left(\xi_{0}, \epsilon\right)=\left\{\xi \in \mathbb{R}^{d}:\left|\xi-\xi_{0}\right|<\epsilon\right\}$. Then

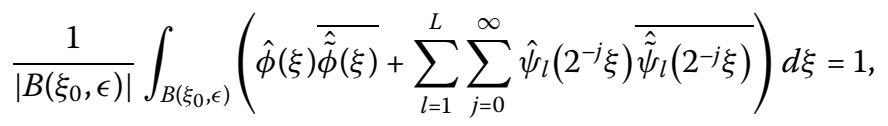

letting $\epsilon \rightarrow 0$ and applying the Lebesgue differentiation theorem, we obtain

$$
\hat{\phi}\left(\xi_{0}\right) \overline{\tilde{\tilde{\phi}}\left(\xi_{0}\right)}+\sum_{l=1}^{L} \sum_{j=0}^{\infty} \hat{\psi}_{l}\left(2^{-j} \xi_{0}\right) \overline{\hat{\tilde{\psi}}_{l}\left(2^{-j} \xi_{0}\right)}=1
$$

For $0 \neq k_{0} \in \mathbb{Z}^{d}$, take $f$ and $g$ such that

$$
\hat{f}\left(\cdot+k_{0}\right)=\frac{\left(1+\|\cdot\|^{2}\right)^{-s / 2} \chi_{B\left(\xi_{0}, \epsilon\right)}}{\sqrt{\left|B\left(\xi_{0}, \epsilon\right)\right|}} \text { and } \quad \hat{g}(\cdot)=\frac{\left(1+\|\cdot\|^{2}\right)^{s / 2} \chi_{B\left(\xi_{0}, \epsilon\right)}}{\sqrt{\left|B\left(\xi_{0}, \epsilon\right)\right|}}
$$

in (3.3), where $0<\epsilon<\frac{1}{2}$. Then

$$
\frac{1}{\left|B\left(\xi_{0}, \epsilon\right)\right|} \int_{B\left(\xi_{0}, \epsilon\right)}\left(\hat{\phi}(\xi) \overline{\tilde{\tilde{\phi}}\left(\xi+k_{0}\right)}+\sum_{l=1}^{L} \sum_{j=0}^{\kappa\left(k_{0}\right)} \hat{\psi}_{l}\left(2^{-j} \xi\right) \overline{\hat{\tilde{\psi}}_{l}\left(2^{-j}\left(\xi+k_{0}\right)\right)}\right) d \xi=0
$$

letting $\epsilon \rightarrow 0$ and applying the Lebesgue differentiation theorem, we obtain

$$
\hat{\phi}\left(\xi_{0}\right) \overline{\hat{\tilde{\phi}}\left(\xi_{0}+k_{0}\right)}+\sum_{l=1}^{L} \sum_{j=0}^{\kappa\left(k_{0}\right)} \hat{\psi}_{l}\left(2^{-j} \xi_{0}\right) \overline{\hat{\tilde{\psi}}_{l}\left(2^{-j}\left(\xi_{0}+k_{0}\right)\right)}=0
$$

By the arbitrariness of $\xi_{0}$ and $k_{0}$, we obtain (3.1).

\section{Competing interests}

The authors declare that they have no competing interests.

\section{Authors' contributions}

All authors contributed equally to the writing of this paper. All authors read and approved the final manuscript.

\section{Acknowledgements}

The article is supported by the National Natural Science Foundation of China (Grant No. 11271037). The authors would like to thank the reviewers for their suggestions which greatly improved the quality of this article.

Received: 11 June 2016 Accepted: 8 November 2016 Published online: 18 November 2016

\section{References}

1. Atreas, N, Melas, A, Stavropoulos, T: Affine dual frames and extension principles. Appl. Comput. Harmon. Anal. 36, 51-62 (2014)

2. Bownik, M: Tight frames of multidimensional wavelets. J. Fourier Anal. Appl. 3, 525-542 (1997)

3. Bownik, M: A characterization of affine dual frames in $L^{2}\left(\mathbb{R}^{n}\right)$. Appl. Comput. Harmon. Anal. 8, 203-221 (2000)

4. Christensen, O, Kim, HO, Kim, RY: On Parseval wavelet frames with two or three generators via the unitary extension principle. Can. Math. Bull. 57, 254-263 (2014)

5. Christensen, O, Kim, HO, Kim, RY: On extensions of wavelet systems to dual pairs of frames. Adv. Comput. Math. 42, 489-503 (2016)

6. Chui, CK, Shi, X: Inequalities of Littlewood-Paley type for frames and wavelets. SIAM J. Math. Anal. 24, 263-277 (1993)

7. Daubechies, I, Han, B, Ron, A, Shen, Z: Framelets, MRA-based constructions of wavelet frames. Appl. Comput. Harmon. Anal. 14, 1-46 (2003)

8. Daubechies, I, Han, B: Pairs of dual wavelet frames from any two refinable functions. Constr. Approx. 20, 325-352 (2004)

9. Han, B: On dual wavelet tight frames. Appl. Comput. Harmon. Anal. 4, 380-413 (1997)

10. Ehler, M: The multiresolution structure of pairs of dual wavelet frames for a pair of Sobolev spaces. Jaen J. Approx. 2 , 193-214 (2010) 
11. Han, B: Pairs of frequency-based nonhomogeneous dual wavelet frames in the distribution space. Appl. Comput. Harmon. Anal. 29, 330-353 (2010)

12. Han, B: Nonhomogeneous wavelet systems in high dimensions. Appl. Comput. Harmon. Anal. 32, 169-196 (2012)

13. Han, B, Shen, Z: Dual wavelet frames and Riesz bases in Sobolev spaces. Constr. Approx. 29, 369-406 (2009)

14. Han, B, Shen, Z: Characterization of Sobolev spaces of arbitrary smoothness using nonstationary tight wavelet frames. Isr. J. Math. 172, 371-398 (2009)

Submit your manuscript to a SpringerOpen ${ }^{\circ}$ journal and benefit from:

- Convenient online submission

Rigorous peer review

- Immediate publication on acceptance

- Open access: articles freely available online

- High visibility within the field

- Retaining the copyright to your article 